\title{
Ein Tag auf dem (Armee-)Sanitätswagen in Murten
}

\author{
L. G. Lorez
}

Korrespondenz: Dr. med. (Sdt) Lukas G. Lorez In der Schappe 157 CH-4144 Arlesheim
Situation: Die militärische Ambulanz wird zur Unterstützung der zivilen Ambulanzen bei der erwarteten Besucherflut anlässlich der Expo 02 und des kantonalen Schwingfestes im Spital Meyriez voll ausgerüstet (2 Mercedes Sprinter) zum Einsatz gestellt.

Um 9.30 Uhr treffen wir mit dem Fahrzeug im Spital ein. Die abzulösende Equipe hatte in der Nacht keinen Einsatz gehabt, der zweite Sanitäter konnte jedoch mit dem ansässigen Krankentransport mitfahren. Er kommt 10 Minuten nach uns von seinem letzten Einsatz dieser Schicht zurück, sehr müde aber mit leuchtenden Augen - diese Nacht ist alles glattgegangen. Wir plaudern noch etwas, auch um unsere Nervosität zu lindern. Insgesamt hoffen wir auf einen Einsatz, den ersten der gesamten Formation. Jeder wünschte jedoch, gewisse Situationen nicht vorzufinden. Bei mir ist es der Herzinfarkt. Als chirurgischer Assistenzarzt ist dies eindeutig kein Krankheitsbild meiner Disziplin. Man trifft sich mit den ansässigen Rettungssanitätern und bespricht das Nötigste.

Der Alarm geht um 10.02 Uhr los, Verdacht auf Herzinfarkt am Schwingfest. Wir rücken aus. Das Schicksal ist manchmal hart. Ausgerechnet ein Herzinfarkt. Ich gehe die Situation auf der Fahrt mit Blaulicht und Martinshorn nochmals mit dem militärischen Rettungssanitäter (Intensivpfleger aus der Ostschweiz) durch. Das Wetter ist gut, die Strassen trocken und so sind wir beinahe schnell am Ort des Geschehens, wäre da nicht ein militärischer Einweisungsposten, der uns fehlleitet. In manchen Köpfen bedeutet $\mathrm{M}$ auf dem Nummernschild = Übung. Bei offenem Fenster mache ich meinem Ärger Luft und bald sind wir dort. Im Samariterzelt liegt ein etwa 58jähriger Metzgermeister mit aufgedunsenem Gesicht, der über Schmerzen in der linken Brust klagt. Es sei nicht der erste Infarkt, der ihn heimsuche. Er leide auch an weiteren Erkrankungen, welche das Körpergewicht von $140 \mathrm{~kg}$ bei $170 \mathrm{~cm}$
Grösse mit sich bringen. Die Notärztin vor Ort hat die Primärversorgung schon vorgenommen. Wir schliessen den Patienten an unser EKG, an die Sauerstoffversorgung usw. an und machen das Fahrzeug abfahrbereit. Die Ehefrau des Patienten fährt natürlich auch mit, womit, mit nun 6 Personen, die 360-kg-Zuladung eindeutig überschritten werden - was für ein Konstrukteur. Der Funkverkehr gelingt uns nicht - ein Empfangsloch? Natel sei Dank gelingt die Verbindung, und der Patient wird ins Spital Meyriez gebracht (im Ernstfall werden alle Natels abgeschaltet). Die Übergabe an die Assistenzärztin (eine Bekannte der Uni) geht reibungslos, der Patient als ehemaliger militärischer Haudegen sehr zufrieden. Wir retablieren und erlernen und erledigen den Schreibkram. Eine kurze Einsatzbesprechung mit den korrigierenden Zielvorgaben, es ist inzwischen 12.45 Uhr, Zeit für eine Pause, aber - es piepst. Der Herzinfarktpatient muss auf eine Intensivstation. Burgdorf ist das nächste Spital seiner Wohngemeinde. Wir überführen den Patienten voll monitorisiert (ein solches Herz kann ja jederzeit die Arbeit einstellen - tat dies aber freundlicherweise nicht).

Endlich die verdiente Pause in einem bekannten Schnellimbisslokal - beinahe hätten wir unser Dach (2,75 m) wegrasiert, also kein DriveThrough. Die Bereitmeldung, auch hier via Natel. Das Essen stehe seit 2 Stunden bereit. Wir treiben unseren Fahrer an. Als gebrannte Kinder stürzen wir das mikrowellenwarme Essen runter - es piepst. 15.30 Uhr, die Schwinger haben schon die 5. Runde erreicht. Einer von ihnen fiel unglücklich auf den Kopf und erleidet eine Halswirbelsäulenverletzung mit Lähmungserscheinungen im rechten Arm. Der Armeeposten winkt uns überfreundlich ein. Diese Verletzung entspricht nun eher meinen Kenntnissen und ich bin bereit, mein Bestes zu geben. Aber auch hier hat die hervorragende Notärztin schon alles erledigt. 
Im Wissen um die Notwendigkeit eines Computertomogrammes bringen wir den Patienten nach Natelabsprache in die Insel. Die Schwester des Patienten kommt mit, und weil der Rettungssanitäter nur Augen für sie hat, muss ich die Geräte und den Patienten beobachten. Auch hier funktioniert die Übergabe reibungslos. Mittlerweile ist Nachtessenszeit und wir hoffen auf eine rasche Rückfahrt. Leider macht uns die Fahrzeugtechnik einen Strich durch die Rechnung und wir tuckern mit $50 \mathrm{~km} / \mathrm{h}$ auf der Autobahn. Nun benötigen wir die Truppe. Das Ersatzfahrzeug trifft zeitgleich in Meyriez ein. Später erfahren wir, dass eine Sicherung nicht korrekt funktionierte - kleine Ursache, grosse Auswirkung. Nun wieder essen so schnell es geht, denn - ja tatsächlich, der nächste Alarm. Wir verlegen eine frischoperierte und intubierte Patientin auf die Intensivstation des Kantonsspitals Freiburg. Die Anästhesistin und ein ansässiger Rettungsfahrer unterstützen uns. Es sind kaum $20 \mathrm{~km}$, jedoch 1000 Kurven. 40 Minuten dauert die Fahrt. Wir betreten die Notfallstation, hypermodern, aber kein Personal, welches uns in Empfang nimmt.

Es braucht drei Anläufe, bis wir eine mobile Sauerstoffflasche an einem Bett abmontieren und die Patientin auf die IPS bringen. Der Empfang ist leider sehr frostig. Zurück im Spital Meyriez sind wir zufrieden, aber müde. Es ist 21.30 Uhr. 12 Stunden Nonstopeinsatz. Die Ablösung kommt. Die ansässigen Rettungsfahrer allerdings machen weiter, sie arbeiten in 96-Stunden-Schichten! 